\title{
Remote Lab Experiments: Opening Possibilities for Distance Learning in Engineering Fields
}

\author{
Oriel A. Herrera ${ }^{1,3}$, Gustavo R. Alves ${ }^{2}$, David Fuller ${ }^{3}$, Roberto G. Aldunate ${ }^{4}$ \\ 1 Informatics Engineering School, Universidad Católica de Temuco. \\ Manuel Montt 56 Temuco, Chile. oherrera@uct.cl \\ 2 Electrical Engineering Department, Instituto Politécnico do Porto, \\ Portugal,gralves@dee.isep.ipp.pt \\ 3 Computer Science Department, Pontificia Universidad Católica de Chile. \\ V. Mackenna 4860, Santiago, Chile.dfuller@ing.puc.cl \\ 4 Applied Research Associates, Inc., Champaign, IL, USA; \\ Department of Civil and Environmental Engineering, University of Illinois \\ at Urbana Champaign, Urbana, IL, USA. aldunate@uiuc.edu
}

\begin{abstract}
Remote experimentation laboratories are systems based on real equipment, allowing students to perform practical work through a computer connected to the internet. In engineering fields lab activities play a fundamental role. Distance learning has not demonstrated good results in engineering fields because traditional lab activities cannot be covered by this paradigm. These activities can be set for one or for a group of students who work from different locations. All these configurations lead to considering a flexible model that covers all possibilities (for an individual or a group). An inter-continental network of remote laboratories supported by both European and Latin American institutions of higher education has been formed. In this network context, a learning collaborative model for students working from different locations has been defined. The first considerations are presented.
\end{abstract}

\section{Introduction}

The technological advances of the last decade have brought many changes to our society. The accelerated development of information technologies (IT) has brought about new learning paradigms in education. One of those paradigms is e-learning. It is the use of network technologies to create, foster, deliver, and facilitate learning, anytime and anywhere. This paradigm has been mainly used in fields where 
practical activities are not required. However, in engineering fields practical activities (labs) are absolutely necessary. Experimentation laboratories must be part of any engineering program. We have been working on remote experimentation laboratories in order to include engineering fields in the e-learning paradigm.

Remote experimentation arises from different motivations:

- It allows teachers to better demonstrate physical concepts during a traditional lecture, by simply connecting to the remote lab and running the experiments;

- It is an economic solution for distance learning courses in engineering fields;

- It allows access to expensive equipment (e.g., an electron microscope) on a 24hour, 7-day basis, giving students the opportunity to use it;

- It allows collaborative work, although this set-up brings additional requirements, especially an interaction model between students. The existence of synchronous and asynchronous communication tools is required.

Once the remote experiment has been implemented, it is necessary to define the methodology that will be used in order for the students to use the experiment in a pedagogical sense. Figure 1 (left side) shows the traditional scenario (present) for a laboratory experiment, where face-to-face interaction plays a fundamental role. On the other hand, a distributed scenario (right side) shows new elements that are necessary for implementing a remote experimentation experience: synchronous and asynchronous interaction, technical issues, time difference, and cultural aspects.

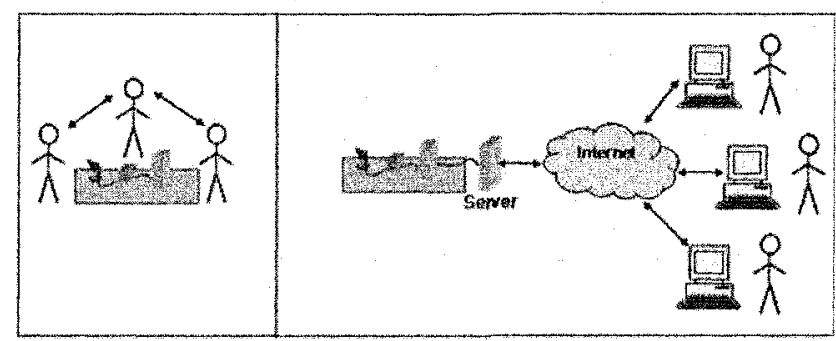

Figure1. Configuration for a typical lab practice (left) and a remote lab practice (right).

This article presents the efforts of an integration project for creating a network of remote experiment labs in engineering fields. The methodology aspects are very important. A collaborative model for groups of students who work from different places is presented. In the rest of the paper section 2 provides some more background on remote experimentation, section 3 presents the network composition and project goals, section 4 highlights the methodology used for remote experiments between students from different universities, and section 5 concludes the paper.

\section{Remote labs background}

The use of Computer Based Learning (CBL), widely associated with similar expressions such as e-learning and distance learning, is widespread among universities. Within engineering, the educational community has felt the need for more powerful combinations: linking educational contents from several sources; 
links from text documents to hands-on modules such as simulations, and finally to real-world experiments [1]. The World Wide Web (WWW) and its associated technologies provided the platform for large-scale implementation of such concepts and ideas, including the free offer of educational materials to the entire engineering community [2]. On the last topic, the first references to making an entire undergraduate lab available through the WWW, date back to the mid 90's. Aktan [3] claims his real-time remote-access control engineering teaching lab to be the first undergraduate lab with complete internet access. Esche [4] describes a more recent undergraduate lab with a strong emphasis on pedagogical issues and enabling technologies. Considering the scenario in Figure 1 (right side), additional questions are raised if the remote experiment calls for collaborative work [5-7]. Since teamwork is one of the most valued aspects of lab practice, such a scheme should benefit from having students from several universities working together to achieve a certain learning objective. In our opinion, cost figures are generally not perceived in the same way by different universities, and thus the number of potential users may diminish substantially. This situation fails to observe the basic principle of inclusion, and so, in our opinion and concerning IT, universities should cooperate on a no-cost basis by sharing e-services among themselves. This idea was at the inception of a proposal to the ALFA II program, entitled Remote Experimentation Network, yielding an inter-university peer-to-peer e-service (RexNet-yippee).

\section{The ALFA-II RexNet-yippee project}

The project goals are to share, harmonize, and spread current skills on remote experimentation. The consortium is formed by two balanced groups from Europe and Latin America, each headed by an Institution of Higher Education with coordination duties. They are: the Polytechnic Institute of Porto (IIPP), the University of Porto (UP), the University of Bremen (UB), the Technical University of Berlin (TUB), the University of Dundee (UD), the Federal University of Santa Catarina (UFSC), the Federal University of Rio Grande do Sul (UFRGS), the Catholic University of Chile (PUC), the Catholic University of Temuco (UCTemuco), the Institute of Technology and Higher Education of Monterrey (ITESM), Mexico. Besides the social interaction, all partners are encouraged to promote the development of new remote experiments and to harmonize the interface to each remote experiment (or lab), namely of those already available [8-12].

\section{Collaborative model for remote experimentation}

In our view, the social involvement of at least one highly motivated player deeply involved in remote experimentation is key to the success of such a peer-to-peer network. It is precisely the human/social factor that ultimately makes the difference: if users know that the system is open to a large community with a high chance of interacting with people from other countries and also that there is a common basis for understanding, namely the subjects addressed by the practical work in a given remote experiment, then motivation increases and the level of skepticism towards a 
remote approach decreases. The combination of higher motivation and lower skepticism undoubtedly increases the educational value of remote experimentation.

A very important role is played by the methodology that will be used for defining the activities associated with a remote experiment. A model that guides the configuration of the main issues to keep in mind is required. These issues are:

- Number of participants: to define if the lab is individual or for a group. For groups we assume the students would work in a distributed scenario.

- Interaction tools: all possible interactions must be considered (students-students, student-tutor, tutor-students, student-contents).

- Other tools: beside interaction tools, many other tools support the activity. The following should be considered: coordination module for accessing the experiment (schedule), discussion forum, text chat, voice chat, videoconference, content management, etc. All these tools could be joined in some Learning Management System (LMS) in order to facilitate the location and use of them.

Also, it is necessary to keep in mind that these issues are directly associated with lab activity. These can be divided from two points of view; one on the contents or materials associated with the lab, and the other on the interaction among participants.

Regarding the course's contents, the following needs to be considered:

a) theoretical guide of the experiment: it considers all necessary theory for understanding and carrying out the experiment;

b) laboratory guide: it specifies the activities that must be performed step by step;

c) experiment help: it includes help from the technology point of view. It should be included in the experiment interface;

d) expected results guide: it specifies the report expected, that is, the result of the experiment (sections, format, delivery instructions, etc.).

The following elements should be considered for the interaction:

a) First interaction: assuming it's the first time the group members work together, an asynchronous interaction must be considered. A discussion forum where each student introduces himself/herself must be set. Also, one or two synchronous sessions with chat and videoconference tools should be considered. The results of this first interaction are the knowledge of the group members, the role and task to be carried out by each student, and the global work schedule.

b) Interaction during the experiment: besides the experiment interaction interface, the synchronous interaction tools must be considered.

This model has been implemented recently for a remote lab in computer science. It involves students from UCTemuco (Chile) and students from ITESM (Mexico). The LMS EDUCA is being used for interaction between students and tutors [13].

\section{Conclusion}

In conclusion, we believe that in addition to the listed benefits and challenges associated with a peer-to-peer network of remote labs, it is possible for others to exist or arise, depending on the type of remote lab in question or on the natural technological advances that often solve old problems and always create new ones. 
New possibilities in distance learning for engineering fields are opened. This approach could be expanded to other fields that require experimentation as an important aspect of the learning process.

\section{Acknowledgements}

The authors acknowledge the sponsorship of the European Commission, EuropeAid, Cooperation Office, through Project ALFA-II-465-A.

\section{References}

1. Cobby, M., Nicol, D., Durrani, T. S., Sandham, W. A., "Teaching Electronic Engineering Via the World Wide Web", Proceedings of the IEE Colloquium on Computer Based Learning in Electronic Education, 1995, pp. 7/1-7/11.

2. Richards, P., "MIT to make nearly all course materials available free on the WWW", 2001, http://web.mit.edu/newsoffice/nr/2001/ocw.html.

3. Aktan, B., Bohus, C. A., Crowl, L. A. and Shor, M. H., "Distance Learning Applied to Control Engineering Laboratories", IEEE Transactions on Education, Vol. 39, n. 3, August 1996, pp. 320-326.

4. Esche, S. K., "On the Integration of Remote Experimentation into Undergraduate Laboratories - Technical Implementation", submitted to International Journal of Instructional Media, 2005.

5. Callaghan, M. J., Harkin, J., Peters, C., McGinnity, T.M., and Maguire, L. P., “A Collaborative Environment for Remote Experimentation", Proceedings of the International Conference on Microelectronic Systems Education, 2003

6. Erbe, H-H., and Bruns, F. W., "Didactical Aspects of Mechatronics Education", Proceedings of the 5th IFAC International Symposium on Intelligent Components and Instruments for Control Applications, Aveiro, Portugal, 2003.

7. Faltin, N., Böhne, A., Tuttas, J., and Wagner, B., "Distributed Team Learning in an Internet-Assisted Laboratory", International Conference on Engineering Education, Manchester, U.K., August 18-21, 2002.

8. Remote Experimentation Laboratory (RexLab), http://rexlab.ufsc.br/, 2005.

9. Remote Electronics Workbench, http://www.fe.up.pt/ jmf/rew, 2005.

10. Remote lab on robotics, automation and control, http://automation.eletro.ufrgs.br/, 2005.

11. Lab@future - a mixed reality web service for Mechatronics, http://lab.artec.unibremen.de/, 2005.

12. Laboratorio Virtual de Robótica Móvil, http://doc.mor.itesm.mx:8181/robot/, 2005 .

13. Courseware platform from the Catholic University of Temuco (January 29, 2006), http://educa.uct.cl, 2006. 